\title{
TRIUNFO DE LA MUERTE DE PETRARCA TRADUCIDO POR JUAN DE COLOMA
}

\author{
M $^{\text {a Pilar Manero Sorolla }}$ \\ Universidad de Barcelona
}

En el entramado de la recepción hispánica del primer petrarquismo en lengua vulgar ${ }^{1}$, resulta superfluo recordar la grandísima popularidad alcanzada por los Triunfos del aretino ${ }^{2}$, en consonancia con el éxito logrado por el libro en la Europa renacentista ${ }^{3}$, comenzando por la propia Italia ${ }^{4}$. La "poé-

1 Para un panorama general con su correspondiente bibliografía remito a mi estudio, Introducición al estudio del Petrarquismo en España, PPU, 1987, pp. 66-73, principalmente.

2 Un estudio particularizado del fenómeno lo ofrece A.J. Cruz, The Trionfi in Spain: Petrarchist Poetics, Translation Theory, and the Castilian Vernaiular in the Sixteenth Century, "Petrarch's Triumphs. Allegory and Spectacle», K. Eisenbichler-AmilCare A. Iannucci (ed.), University of Toronto, Italian Studies, 4, Toronto, Dovehouse Editions, 1990, pp. 259-268.

' Ver la relación fundamental de E. H. WILKINS, A General survey of Renaissance Petrarihism, "Comparative Literature", 4 (1950), pp. 327-341. También la más reciente debida a F. Guardiani, The Literary Impact of the Trionfi in the Renaissance, "Petrarch's Triumphs. Allegory and Spectacle" cit., pp. 259-268. En relación a petrarquismos más específicos, merecen citarse en este sentido los estudios de E. Pellegrin, Manuscrits de Pétrarque dans les bibliothèques de France, Padova, Antenore, 1960; F. SimONE, Note sulla fortuna de Petraria in Francia nella prima metà del Cinquecento, "Giornale Storico della Letteratura Italiana", 127 (1950), pp. 1-59; ID., Il Petraria e la cultura francese, "Studi francesi", XIV (1970), pp. 201-205 y 403-417, y D. CeCCHetTI, Il petraribismo in Francia, Torino, G. GIAPPINELLI, 1970, por cuanto concierne al petrarquismo francés. En lo referente al petrarquismo inglés resulta ilustrativa la obra de N. MaNN, Petrarch manuscripts in the British Isles, Padova, Antenore, 1976. Para otras latitudes europeas, dan cuenta asimismo de la fortuna alcanzada por los Triunfos, G. C. Rossı, La tradizione del Petrarchismo nella Letteratura Portoguese, "Atti dei Convegni Lincei", cit.. pp. 73-102 y A. Sotrill, I codici del Petrarca nella Germania occidentale, Padova, Antenore, 1971, como, asimismo, los diversos catálogos aparecidos en Italia Medioevale e Umanistica, 1967-72 y 1975.

4 Ilustra el fenómeno el ya clásico estudio de C. Dionisotri, Fortuna del Petrarca nel Quattrocento, «Italia Medioevale e Umanistica», XVII (1974), pp. 61-113. También los artículos de E. H. WILKINS, The separate fifteenth-century editions of the Triumphs of Petrarch, "The 
tica triunfal" de la obra, en parte medievalizante y común a Dante y Boccaccios, pero con una figuración infinitamente más accesible y más próxima a la sensibilidad humanística" que las abstracciones morales expresadas por las alegorías de la Dizina Commedia, resultaba apropiada y atractiva para un público lector, no enteramente despegado del pensamiento medieval, pero estéticamente abierto al espectáculo y a la magnificencia de las pautas artísticas prerrenacentistas contenidas en los Trionfi.

Incluso el argumento central, tan petrarquesco, y el mensaje final del libro se prestaban a hacer de él una cibra puente entre dos tendencias estéticas e ideológicas. La disposición misma de la alegoría, desde la perspectiva de su significativo desenlace, presentando a la Eternidad, último de los Triunfos, vencedora del Tiempo, el Tiempo de la Fama, ésta de la Muerte y la Muerte, a su vez, de la Castidad, victoriosa del Amor, Triunfo inicial, evidenciaba, al menos después de la reestructuración final del escrito que Petrarca rubricó el 12 de febrero de 1374 , la última voluntad poética del autor y su creencia en la vida del más allá: la eternidad, colocada por encima de los valores terrenos, subrayados con marcado acento renacentista en el Triunfo de la Fama y potenciados por el mismo escritor en épocas anteriores de su existencia más alejadas de su propia muerte, acaecida en Arquà el 19 de julio del mismo año de 1374 . Desenlace, disposición y mensaje que, junto a la alegoría continuada, como forma fundamental de expresión, y la retórica enumerativa de alusiones eruditas, de ambivalencia cristiano-pagana, coadyuvaron a crear la "factura" humanística de la obra ${ }^{8}$ y a explicar por qué la recepción de la misma se registrase con anterioridad a la del Canzoniere y, en parte, paralelamente a la del Petrarca latino, en la doble línea de expansión característica del petrarquismo medieval, y aun renacentista, de la que el petrarquismo español' es buen exponente.

Library Quarterly", XII (1942), pp. 7.48-751 y G. GufrRrINI, Per uno studio sulla diffusione manosiritta dei Trionfi di Petraria nella Roma del XV secolo, "Rassegna della Letteratura Italiana", 1983 (86), pp. 85-97.

'Lo explica pormenorizadamente A. S. Bernarid), Triumphal Poetry: Dante, Petrarih and Bociaciio, "Petrarch's Triumphs. Allegory and Spectacle" cit., pp. 33-47.

"G. Erasmi, Petrarch's Trionfi: The poetics of Humanism", Petrarib's Triumphs. Allegory and Spectacle cit., pp. 161-176.

Ver E. H. WILKINS, Life of Petrarch, The University of Chicago, 1961. Cito por la traducción italiana de R. Cesarini, Vita del Petraria e La formazione del *Canzoniere», Milano, Feltrinelli, 1970, p. 315.

${ }^{8}$ C. F. Goffis, Originalità dei «Trionfi», Firenze, La Nuova Italia, 1952, p. 51.

" Para mayores particularidades sobre esta cuestión y síntesis bibliográfica al respecto remito a mi Introducioión al estudio del petrarquismo en España cit., pp. 13-15 y A. DEYERMOND, The Double Petrarchism of Medieval Spain, "Journal of the Institute of Romance Studies", 1 (1992), pp. 69-85. 
La impronta de los Triunfos se manifiesta así como primera expresión del petrarquismo vulgar en la literatura española ${ }^{11}$ entre los poetas del siglo XV. Conocidos por Juan de Mena ${ }^{11}$ y Jorge Manrique'.', su huella se patentiza de manera más rotunda en la obra del Marqués de Santillana. influjo que ha sido subrayado, tanto por lo que se refiere a las variadas reminiscencias detectadas en El Sueño e Infierno de Enamorados ${ }^{13}$, como de manera más evidente y directa en la Comedieta de Pong $a^{1+4}$, en el cortejo de la Fortuna con su forma procesional, tan característica, del Triunfo de Amor, de la Muerte y de la Fama. Y, especialmente, por ser el Triunfo de Amor petrarquesco modelo alegórico del Triunfete del Marqués ${ }^{15}$, con posibles futuras variantes quinientistas, como es el caso del Triunfo de loj Celos del peruano Diego Dávalos de Figueroa en su Miscelánea Austral" ". Repercusión alegórica y plástica que, readaptada estéticamente hallará su expresión barroca en el siglo XVII en un proceso de divinización que, sin embargo, comienza en el filo del siglo XV y XVI con los Siete triunfos de las siete virtudes de Alonso Hernández ${ }^{17}$, el Aucto de los Triunfos de Petrarca a lo divino del Códice de

11' A. J. CRuz, The Trionfi in Spain: Petrarchist Poetics, Translation Theory and the Castilian Vernacular in the Sixteenth Century, "Petrarch's Triumphs. Allegory and Spectacle" (it., pp. 307-204. Evidencia la difusión manuscrita M. VILLAR RuBIO, Códices petrarquesios en España, Tesis doctoral dirigida por el profesor F. Rico, Universidad Autónoma de Barcelona, 1988-Padova, Antenore, en prensa.

"i Ver M. R. Lid)a, Juan de Mena poeta del Prerrenaimiento español, México, El Colegio de México, 1950, p. 353. También R. LAPESA, Poesia de cianizonero y poesía italianizante", De la Edad Media a nuestros dias, Madrid, Gredos, 1967, p. 148.

1: Ver G. CanglotTi, Las Coplas de Manrique tra medioevo e Unianesimo, Bologna, Casa Editrice Riccardo Patron, 1964, p. 147 y el clásico artículo de J. VINCI, The Petrarchan Sources of Jorge Manrique, "Italica», XIV, pp. 314-328.

13 Ver M. Durán, "Prólogo" a las Poesías Completas del Marqués de Santillana, Madrid, Castalia, 1975, I, p. 18.

1. Ver el clásico estudio del Conde de Puymaigre, La cour littéraire de Don Juan II roy de Castille, Paris, Librairie A. Franck, 1875, I, p. 28.

is Ver R. LAPESA, La obra literaria del Marqués de Santillana, Madrid, Ínsula, 1957, p. 114. El influjo, en este sentido, lo señala ya A. Farinfillo en Petraria in Ispagna, Italia e Spagna, Torino, Fratelli Bocca-Editori, 1928, I, p. 39. Pero ver también a este propósito el artículo de J. Jimeno Casalduero, El Triumphete de Amor del Marqués de Santillana: fuentes, composición y significado, "Nueva Revista de Filología Hispánica", XXVIII (1979), pp. 318327 y el más reciente de G. Caravaggi, Petrarch in Castile in the Fifteenth Century: The Triumphete de Amor by the Marquis of Santillana, "Petrarch's Triumphs" cit., pp. 291-306.

16 Analiza el mismo A. De Colmbí-Monguió, Petrarquismo peruano. Diego Dávalos y Figueroa y la poesia de la Miscelánea Austral, London, Tamesis Books Limited, 1985, pp. 196206.

Ver M. Menéndez y Pelayo, Antología de poetas líricos castellanos desde la formación del lenguaje hasta nuestros dias, Madrid, Vda. de Hernando, 1890-1908, vol. III, p. 118. 
Autos Viejos ${ }^{1 \mathrm{x}}$, para continuar manifestándose de manera más vaga e imprecisa, en los Triunfos de Maria de Martín de Ampies" ", los Veinte Triunfos de Vasco Díaz de Fregenal ${ }^{20}$ o en los Triunfos Morales y emblemáticos de Francisco de Guzmán y en los Triunfos del amor de Dios de Fray Juan de los Ángeles ${ }^{21}$. Sin olvidar los Triunfos divinos de Lope de Vega en los que, en cambio, la huella de Petrarca resulta notable ${ }^{22}$, incluso desde el punto de vista estructural.

Entre las imitaciones cuatrocentistas del modelo "triunfal" y las barrocas a lo divino constatamos la floración de las traducciones en lengua castellana ${ }^{2}$. Las de los Trionfi preceden asimismo, a las del Canzoniere ${ }^{2.4}$ y, en parte, se ofrecen paralelas a las de las obras latinas de Petrarca que se inician en 1450, año en que el bachiller Hernando de Talavera realiza una traducción de las Invective contra medicum con el título de Reprehensiones y denuestos contra un medico rudo y parlero ${ }^{25}$ y culminan en la primera mitad del quinientos. Las primeras, en vulgar, superando el límite y atravesando el siglo (1512-1592): ambas, Trionfi, Canzoniere, destacadas en el contexto de las traducciones españolas que de la literatura italiana se realizaron durante el Siglo de Oro ${ }^{26}$.

Poseemos algunos vestigios manuscritos de traducciones castellanas de

1* Ver M. Lentzen, Zur Rezeption Petrarcas in Spanien, Petraria 1304-1374. Beiträge zu W'erk und Wirkung, Frankfurt am Main, Vittorio Klostermann, 1975, pp. 139-155.

19 Editadas en Zaragoza en 1495 y reeditados en facsímil por A. PÉrez. GómEZ en Valencia, "...la fonte que mana y corre...", 1945.

2n Ms. de BNM, reproducido por la Junta Técnica de Archivos, Bibliotecas y Museos, Madrid, 1945.

${ }^{21}$ Los primeros fechados en 1587 y en 1598 los segundos. B. WARIDROPPER los estudia en La historia de la poesía a lo divino en la cristiandad occidental, Madrid, Revista de Occidente, 1958, pp. 272 y ss.

22 Ver M. P. Manero Sorolla, Las traduciones castellanas quinientistas de la obra de Petrarca, Introducción al estudio del petrarquismo en España cit., pp. 140-159.

2i F. Meregallo estudia las primeras en Sulle prime traduzioni spagnole di sonetti del Petrarca, Traduzione e tradizione europea del Petrarca. "Atti del III Convegno sui problemi della traduzione letteraria», Monselice, 9 giugno 1974, Padova, Antenore, 1975.

24 Ver F. Rico, Cuatro palabras sobre Petrarca en España", "Convegno Internazionale Francesco Petrarca» (Roma-Arquà 24-27 aprile 1974), "Atti dei Convegni Lincei»10, Roma, Accademia Nazionale dei Lincei, 1976, p. 54.

${ }^{25}$ Para una fechación y confrontación en la aparición de las distintas traducciones señaladas ver mi estudio Las traducciones castellanas quinientistas de la obra de Petraria en $\mathrm{mi}$ Introducción cit., pp. 153-156.

26 Esboza su historia y proporciona relación de los libros más significativos traducidos del italiano al español, E. SECO, Historia de las traducciones literarias del italiano al español durante el Siglo de Oro (Influencias), "Cuadernos para Investigación de la Literatura Hispánica», 13 (1990), pp. $41-97$. 
los Trionf $i^{27}$ anteriores a la de Antonio de Obregón, la primera completa, editada por vez primera en Logroño en el año de $1512^{28}$, pero con reediciones escalonadas a lo largo de la primera mitad del quinientos (1526, 1532, $1542)^{29}$. En el inicio de la segunda, concretamente en 1554, Hernando de Hozes publicó otra traducción de los Trionf $i^{30}$ con segunda edición en $1581^{31}$. Si la primera de Obregón se servía del tradicional verso de arte mayor castellano y medievalizante, la de Hozes, efectuada más allá de la introducción del verso endecasílabo en la lírica española por Boscán y Garcilaso, se hacía «en la medida y número de versos que tienen en el toscano". Y junto a éstas tendríamos que hacer mención de las traducciones parciales como la del Trionfo d'Amor de Alvar Gómez de Ciudad Real ${ }^{32}$ y las inéditas de Luis Zapata (?), Jerónimo de Urrea (1549) y Alcocer (1550), conservadas en la $\mathrm{BNM}^{33}$.

La traducción del Triunfo de la Muerte de Juan de Coloma aparece más allá de la época de apogeo en la recepción de los Triunfos en Europa y en España, pero antes que la primera, aunque tardía, traducción castellana del Canzoniere ${ }^{34}$, la de Salomón Usque (1591), y coincidiendo con la publicación

27 Ver en este sentido el antiguo estudio de B. SANvisenti, I primi influssi di Dante, del Petrarca e del Boccaccio sulla Letteratura Spagnola, Milano, Ulrico Hoepli 1902, p. 417, como asimismo el clásico de A. FARINelli, Sulla fortuna del Petrarca in Spagna nel Quattrocento, Torino, Bocca, 1904, p. 116.

28 F. PetrarCa, con los seys triunfos de toscano sacados en castellano con el comento que sobrellos se bizo, Logroño, Arnau Guillén de Brocar, 1512.

${ }^{29}$ Guarda de todas ellas ejemplares la BNM: R-11730 (Sevilla, 1526); R-29 (Sevilla, 1532); R-12172 (Valladolid, 1542).

${ }^{30}$ Los Triumphos de Francisco Petrarca, ahora nueuamente traduzidos en lengua Castellana, en la medida y numero de versos que tiene en el Toscano y con nueva glosa, Medina del Campo, Guillermo de Millis, 1554.

31 En la BNM (ms. 3687) existe también un códice que contiene los Triumphos en una redacción de hacia 1549 distinta de la citada.

32 Recogida por los códices manuscritos de Ixar y Gallardo y posteriormente impresa acompañando a unas cuantas ediciones de la Diana de Jorge de Montemayor, concretamente las de Cuenca, 1561; Valladolid, 1561; Alcalá, 1564; Lisboa, 1565; Pamplona, 1578; Amberes, 1580; Venecia, 1585; Madrid, 1586 y 1595; Valencia, 1602; Madrid, 1602 y 1622 y Lisboa, 1624. Cuenta con un pormenorizado estudio de G. C. Rossi, Una traduzione cinquecentesca spagnola del Trionfo d'Amore, "Convivium", V (1959), pp. 40-50. Ver también de J. M² Azaceta, Petrarca traducido por Alvar Gómez. Nuevos datos para el estudio del influjo del poeta italiano en nuestra lírica renacentista, El Cancionero de Gallardo, Madrid, CSIC, 1962, pp. 42-48. Ver también el estudio de F. LOPEz Estrada a su edición de la Diana de Montemayor, Madrid, 1967, pp. LXXXVII-CII.

33 Ver el estudio de F. Rico, El destierro del verso agudo, "Homenaje a José Manuel Blecua ofrecido por sus discípulos, colegas y amigos», Madrid, Gredos, 1983, p. 533.

34 De los Sonetos, Canciones, Mandriales y Sextinas del gran Poeta y Orador Francisco Petrarca, traduzidos de toscano por Salusque Lusitano. Parte Primera... Venecia, Nicolao 
de Hernando de Hozes (1554), pues forma parte del Cancionero general de obras nuevas nunca hasta aora impresas assi por ell arte española como por la toscana, publicado en Zaragoza, por Esteban de Nájera, también en $1554^{39}$. Su autor, conde de Elda, debió también nacer a principios del siglo XVI, muriendo en $1586^{36}$, después de ostentar el cargo de alcaide del castillo de Alicante (1573-1576). Su obra poética parece haber sido estimada por sus contemporáneos: Jerónimo Jiménez de Urrea la elogia en su traducción del Orlando Furioso (1549) ${ }^{37}$, mientras que Hernando de Hozes cita a Coloma como autoridad en el prólogo de su propia traducción de I Trionfi. Le nombran también poetas menores como Zapata en su Carlo famoso (1566) $)^{38}$ y Gregorio Hernández de Velasco en el Parergon de su traducción De partu Virginis (1771) ${ }^{39}$. Más conocido es el elogio que Cervantes le dispensa en el Canto de Caliope:
¡O tu don Iuan Coloma, en cuyo seno tanta gracia del cielo se ha encerrado, que a la embidia pusiste en duro freno y en la fama mil lenguas has criado, con que del gentil Tajo al fertil Reno tu nombre y tu valor va levantado! Tu, Conde de Elda, en todo tan dichoso, hazes el Turia mas qu'el Po famoso. ${ }^{40}$

Bevilaqua, 1567. Ver al respecto: M. P. MANERo SOROLA, La primera traducción de las Rime de Petrarca en lengua castellana: los sonetos, canciones, mandriales y sextinas del gran poeta y orador Francisco Petrarca de Salomón Usque, «Homenaje al profesor Antonio Vilanova», Universidad de Barcelona, 1989, pp. 377-391.

$3 s$ Del Cancionero se conserva un único ejemplar en la Werner-Herzog Bibliotek de Wolfenbüttel estudiado y transcrito por F. WolF, El Cancionero de 1554, «Sitzungsberichte der Kaiserlichen Akademie der Wissenchaften. Phil. Hist. Klasse» Band X, Wien, 1853, pp. 153-204; A. MOREL-FATIO, L'Espagne au XVI et XVII siecle. Documents bistoriques et litteraires, Heilbronn, Henniger Frères, 1878, pp. 491-602; C. MichaElis DE VAsConCellos, Zum Cancionero General de Nagera, *Zeitschrift für romanishe Philologie», V (1881), pp. 77 79; J. B. W. CRAWFORD, Notes on the sonnets in the spanish Cancionero general of 1554 , «Romanic Review», VII (1916), pp. 328-338. Recientemente ha sido editado por C. Claveria, Barcelona, Delstre's, 1993, ed. por la que cito.

36 B. E. EnTEnza DE SOlARE, Datos para la biografia de Don Juan Coloma, «Filología» (Buenos Aires), XIII (1968-1969), pp. 165-173.

${ }^{37}$ Canto XLI, octava 106, Anveres, Martín Nucio, 1549, fo. 215 v. Ver B. E. EnTenzA DE SOLARE, p. 165.

38 Canto XXXVIII, Valencia, 1566, fo. 204 r-v. Citado por B. E. Entenza de Solare, p. 166.

39 Parnaso español, Madrid, 1771, V, p. 166.

40 La Galatea, VI, 98. Cito por la ed. de J. B. Avalle-Arce, Madrid, Espasa Calpe, 1987, pp. 454-455. 
Conservamos un número considerable de poemas suyos ${ }^{41}$, la mayoría de ellos contenidos en el mentado Cancionero general de 1554 que presenta treinta y cinco composiciones en metros españoles e italianos, entre ellos el Triunfo de la muerte en quintillas dobles, traducción del de Petrarca, que abre las composiciones del Cancionero. Este, en verdad, quiere reflejar desde el mismo título, al propio tiempo que un vínculo con el otro Cancionero general de $1511^{42}$, la novedad que Esteban de Nájera, el editor, introduce como distintivo: las obras nuevas nunca hasta aora impressas por ell arte española en metros tradicionales, de autores varios ${ }^{43}$, que encabezan la colección y, en especial, las todavía más novedosas por el arte toscana, ordenadas en la segunda parte, debidas a Hurtado de Mendoza y al propio Coloma, principalmente, quien, sin embargo, no se acoge al metro endecasílabo ni al estrofismo italianizante en su traducción del petrarquesco Triunfo de la Muerte.

La aclimatación a la lengua poética castellana de los metros venidos de Italia no fue tarea fácil ni se llevó a cabo, de modo satisfactorio, al uníso$\mathrm{no}^{44}$. Precisamente, las varias traducciones de los Trionfi al castellano realizadas entre 1512 y 1555 nos evidencian los cambios de criterio en relación a la poética petrarquista, a la teoría de la traducción y al valor de la lengua vernácula «al evolucionar dichas traducciones desde el estilo cancioneril medieval hasta las nuevas formas italianizantes ${ }^{45}$. Pero, aunque la frontera de los tanteos y del destierro del verso agudo parece rebasada en $1554^{46}$, fecha, por lo demás, de publicación de la mentada traducción completa de los mismos Triunfos petrarquescos, en endecasílabos ordenados en tercetos, debida a Hernando de Hozes, Juan de Coloma, quien posiblemente tradujese su Triunfo de la muerte con anterioridad a estas fechas, se decanta por el metro antiguo; él que, sin embargo, en el mismo Cancionero general de obras nuevas, encabeza «las obras que van por el arte toscana», alguna de ellas, como la Egloga de tres pastores, estructurada en tercetos: justamente la estrofa de los Triunfos en versión original.

${ }^{41}$ Ver el artículo cit. de B. E. ENTENZA DE SOLARE, pp. 166-167 para el inventario de los conocidos.

${ }^{42}$ Cancionero General, recopilado por Hernando del Castillo, Valencia, 1511; ed. facsímil de A. Rodriguez MoNino Madrid, RAE, 1958. Suplemento del Cancionero General, ed. de A. Rodriguez Moñıno, Valencia, Castalia, 1959.

${ }^{43}$ El propio Coloma, Boscán, Pedro de Guzmán, Juan de Mendoça, Gualvez, Luis de Haro, Luis de Narváez, Gabriel, Puertocarrero, Jerónimo de Urrea, entre otros.

44 Ver, en general, el cit. estudio de F. Rico, El destierro del verso agudo, en el que se ilustra igualmente la trayectoria y cronología de la introducción, aclimatación y triunfo de la métrica italianizante en la poesía española renacentista.

${ }^{45}$ Ver el estudio cit. de A.J. CRUz, p. 307, en especial.

${ }^{46}$ F. RICO, El destierro, pp. 528-529. 
Es posible que en esta decantación de Coloma por metro y estrofismo haya tenido que ver el modelo de la traducción realizada anteriormente por Obregón (1512) y, asimismo, el del Trionfo d'amor de Alvar Gómez ${ }^{47}$, también en octosílabos y quintillas dobles. Aunque, aparte de estas coincidencias, ambas traducciones, concretamente la del Triunfo de Muerte de Obregón y Coloma, difieran notablemente en otros aspectos.

La primera, completa, sigue, como es sabido, la edición italiana más divulgada de los Trionfi en el primer Renacimiento, anotada extensamente en 1475 por Bernardo da Pietro Lapino da Montalcino, más conocido como Bernardo Glicino o Illicino ${ }^{48}$, que asimismo influyó en todos los comentaristas posteriores de la obra, pero anotación que, sin embargo, ya no está sólo en la base de la versión de Hozes ${ }^{49}$, y de la que, precisamente, prescinde Coloma.

De hecho, el problema inicial y básico a tener en cuenta, más que en los comentarios, radica en la disparidad de las distintas versiones del original petrarquesco, ya impresas, ya manuscritas, y arranca del fragmentarismo en el que Petrarca fue construyendo los distintos Triunfos, de los que dejó escritas varias redacciones con ordenación diferente de capítulos: de ahí la discrepancia de los manuscritos y aun de los autógrafos conserva$\operatorname{dos}^{50}$, discrepancia que no desconocían los primeros traductores españoles ${ }^{51}$.

La traducción del Triunfo de la Muerte de Juan de Coloma, tal como aparece en el Cancionero General de obras nuevas (1554), no contiene prólogo o declaración ni tampoco presenta comentario o glosa que intente explicar

47 Aunque ignoramos la fecha exacta de la traducción de Alvar Gómez, necesariamente fue anterior a la de Coloma, pues según J. M. Azáceta, su editor moderno (Cancionero de Gallardo, ed. cit., pp. 98-151), Gómez murió antes de 1538.

48 Opera del preclarisimo Poeta Miser Francesco Petrarca con li commenti sopra li Triumphi i Sonetti: Canzoni historiate e nuovamente corrette per Miser Nicolo Peranzone con molte acute e eccellente additione. Miser Bernardo Lycinio sopra li Triumphi. Miser Antonio da Tempo. Hieronymo Alesandrino sopra Sonetti e Canzoni, Venetia, Bartholomeo de Zani de Portese, 1503. Cito siempre por esta edición.

49 "Y así mismo le puse nuevo commento, no tan breue como el de Alexandro Vellutello, ni tan largo en muchas cosas, como el de Bernardo Illicino, sino tomando a pedaços de entrambos, quitando algo de lo que parescía superfluo y añadiendo lo que en $\mathrm{mi}$ juizio era muy necesario*, Hernando de Hozes, Triunfos, ed. de 1581, fo. i, por la que cito.

so C. APPEL estableció en 1901 un texto crítico de la obra: Die Triumphe Francesco Petrarcas. In kritischen Texte herausgegeben. Halle, Max Niemeyer, 1901. Es la edición que suelen seguir los editores modernos en cuanto a texto se refiere; no así en la ordenación de los capítulos para lo que prevalece el criterio canonizado por Bembo en su edición aldina de 1501, más fiel a la última reestructuración de Petrarca antes de su muerte.

s1 Obregón alude al hecho en el prólogo de su propia traducción cit., fo. 2 (ed. de 1541, por la que cito). Hozes en la suya, también cit., compara en este sentido, la distinta ordenación de Bernardo Illicino y Alexandro Vellutello: fos. 179 r. y v., de la ed. cit. 
el texto traducido. Sin división en dos capítulos, según la propia disposición petrarquesca y como generalmente se ha presentado este Triunfo desde antiguo en las versiones originales italianas así como en las traducciones castellanas de Obregón y Hozes, consta de 630 versos distribuidos en 63 quintillas dobles. Excede, por tanto, en 102 versos al texto italiano antiguo de Illicino, que consta de 428 versos. Por lo tanto, y aunque tengamos en cuenta que éstos son de 11 sílabas frente a las 8 del verso usado por Coloma, con todo la traducción de éste es más extensa que el original, lo cual por si sólo no tiene que indicar, necesariamente, que sea una traducción óptima ${ }^{52}$.

Juan de Coloma, como con anterioridad practicó Antonio de Obregón respecto del original italiano, realiza una traducción relativamente ad sensum con concesiones a la réplica literal ad verbum ${ }^{53}$. Ésta, prácticamente, coincide en extensión con la de su predecesor, que tan sólo la sobrepasa en una quintilla doble. Con él también comparte, determinados ambos por la poética cancioneril aneja a la métrica castellana medievalizante, con el verso octosilábico y la rima oxítona, predominante, la tendencia a transformar en abstracciones el mundo poético petrarquesco, consustancialmente figurativo y polisémico. Aunque no siempre coincidan ambos traductores en los lugares transformados ni en las soluciones, formas y modos de la transformación.

Así, por ejemplo, traducen ambos, los tercetos finales, emblemáticos, del tradicionalmente primer capítulo del Triunfo de la Muerte petrarquesco, que describen la idealizada, a la vez que humana, figura de Laura muerta con la preeminencia que tienen siempre en la poesía del aretino los elementos visuales, que la fórmula de traslación que ofrecen nuestros traductores declinan o transforman en proporción y manera distintas:

Poi que desposto il pianto e la paura

Pur al vel volto era ciaschuna intenta, Et per desperation facta secura;

Non come fiamma, che per forza e spenta;

$\mathrm{Ma}$ che per se medesima si consume;

Se n'ando in pace l'anima contenta

52 Recordemos que, según las teorías de G. P. NORTON (Humanist Foundation of Translation Theory (1400-1450): A Study in the Dynamics of the Word, "Canadian Review of Comparative Literature», 2 (1981), p. 176): "Most good translations are longer than the original text and the words of the source language suffer, in transmission to the target language, an accretion of substance».

\$3 La terminología forma parte del primer tratado renacentista sobre la traducción, De Interpretatione recta (1426), de L. BRUNI, cit. por G. P. NORTON, estudio cit., p. 189. 
A guisa d'vn soave, e chiaro lume; Cui nutrimento a poco a poco manca; Tenendo al fin il suo vsato costume; Pallida no ma più che neve bianca Che senza vento in vn bel colle fiocchi; Parea posar, come persona stanca. Quasi vn dolce dormir ne soi begliocchi Essendo'l spirto gia da lei diuiso Era'quel; che morir chiaman sciocchi: Morte bella parea nel suo bel viso.

(Ed. cit. de Illicino, fo. LVII.v.)

El miedo y lamentación depuesto con amargura mirauan su perfección y por desesperación era cada cual segura.

No como llama amatada por fuerça ni por tormenta: mas por si misma acabada se partio muy bien guiada en paz el alma contenta.

Era entonces remedando muy suave y clara lumbre a quien virtud va saltando y poco a poco acabando llegando al fin su costumbre amarilla no por cierto; mas que la nieve blanca: que sin viento cuaja en puerto: y su reposo y concierto: como quien cansa y estanca.

Quasi muy dulce dormir en sus ojos parescia despues de se diuidir alma y cuerpo en el partir paresciendo que dormia espesa de aquesta suerte llama el vulgo mentiroso 
morir por ser trago fuerte y estatua linda la muerte en su rostro muy hermoso.

(Traducción de Obregón, ed. cit., fos. Ixjiji.r-lxjx.v.)

Después qu'el llanto amansado, todos, por desconfiar, se hauían assegurado y el espíritu ensalçado estaua donde ha d'estar, que salió de la manera que acabándose la cera desaparesce la lumbre dando fin a su costumbre, que de dar claridad era.

El cuerpo muerto yazía y el gesto no lo mostraua, que qual la nieue se vía y mucho más parescía que dormía y descansaua. En sus lindos oios era vn dormir la muerte fiera (de muchos ansí llamada), donde estaua tan mudada que nunca le paresciera.

(Traducción de Coloma en ed. cit., p. 11.)

En efecto, mientras que en relación al original italiano, Obregón mantiene, relativamente, la traducción ad verbum, Coloma sigue, de forma general, el sentido, tendiendo habitualmente, inclusive en el momento de tentar la traslación de los elementos figurados, a eliminar palabras, agregando otras que se sobreentienden o pueden sobreentenderse en el original y que él explicita. Así sucede, por ejemplo, con la imagen de la llama apagada (fiamma spenta) con la que Petrarca compara el alma de Laura y a la que está unida la de la luz (lume) acompañada de adjetivos tan relevantes y evocadores del sugerente mundo petrarquesco como suave y clara (soave e chiaro). Obregón conserva las palabras clave (llama, suave y clara lumbre), amplificando el discurso, que Petrarca reduce a un terceto (Non come fiamma...lume), a diez versos octosílabos (No como llama amatada...costumbre), manteniendo 
igualmente ciertas estructuras morfosintácticas como la comparación negativa (Non come fiamma...; No como llama...) y traduciendo prácticamente, palabra por palabra, algún verso (Se n'ando in pace l'anima contenta...; se partió en paz el alma contenta), introduciento, eso sí, vocablos desarmónicos al contexto (tormenta), o pesados gerundios (remedando, saltando, acabando), impropios del evanescente mundo petrarquesco original. Coloma, infinitamente más libre en su traslación, invierte elementos y prescinde de la coincidencia léxica, el calco sintáctico o el seguimiento retórico. Transforma por esta vía, en el discurrir figurativo, la imagen de la llama, exenta de la comparación retórica del texto original, y de su correlativa, la luz, en la afín, pero menos petrarquesca, de la cera unida a la lumbre. Sobreentiende en ello, como se trasluce por la sinécdoque que emplea, la imagen de la vela cuya cera se consume (y con ella se extingue la llama), corriente en la poesía medieval para describir la vida que se acaba; fórmula que, precisamente, Petrarca evita tanto en la materia como en la forma, conservando sólo el vestigio final: llama, el más sustancial a su mundo poético y el más idóneo al punto culminante que estaba describiendo: el tránsito sublimado de Laura.

Algo semejante ocurre a continuación en la descripción del cadáver de la amada que Petrarca magnifica en la victoria de la belleza de Laura sobre la muerte; o la transfiguración de ésta, en su materialidad corpórea específica, en muerte hermosa, tal como rubrica el último verso del primer capítulo del Triunfo: Morte bella parea nel suo bel viso. Indudablemente, se trata de una de las cimas del arte poético petrarquesco y, por ello mismo, pasaje delicado para el traductor. Obregón, sumiso al sentido y aun a la letra del texto original, pero falto de la adecuada sensibilidad poética que exigía el pasaje, traduce, de manera relativamente fiel, unos versos en extremo comprometedores. La inmaculada palidez de Laura, que Petrarca, lapidariamente, compara en competencia con la nieve a la que aquélla aventaja (Pallida no ma piú che neve bianca) es coloreada (amarilla) por el primer traductor y negada trivialmente su anterior calificación (no por cierto): primer miembro de una comparación que queda idéntica para el resto del conjunto (mas que la nieve blanca), excepción hecha del adjetivo bel que el español omite al final (Che senza vento in un bel colle fiochi; que sin viento cuaja en puerto). Adjetivo epíteto del que seguirá prescindiendo en begliocchi (ojos), morte bella (muerte), bel viso (rostro), sin captar el obsesivo norte de belleza que la extrema redundancia por repetición del adjetivo (por lo demás siempre redundante en textos petrarquescos) pretendía suscitar. Contrariamente, ampliará la enumeración de elementos que no figuran en el original, intrascendentes a la descripción (vulgo mentiroso, aquesta suerte, trago fuerte) y ajenos al mundo de Laura. Más ajena todavía la versión de Coloma: réplica ad sensum que prescinde o simplifica los elementos figurativos y retóricos del texto original (exceptuando parte de la comparación del rostro de la bella con la nieve) 
pero que, en cambio, amplifica la parte narratava del masmo, añadiendo versos con información complementaria o de contexto (el ieferpo muerto yazia) o aclaraciones superfluas (de muibus ansi llamada) para expresar una situación que Petrarca, poéticamente, condensa y esencializa, sin prescindir por ello de la figuración, antes bien, sirviéndose de ellia.

La traducción de Coloma ostenta, de hecho, getneralmente y por sistema, las tendencias señaladas. Conformada según la métrica y el estilo cancioneril, difiere, sin embargo, del modelo ane erior de Obregón, sujeto al mismo código pero literalmente más fiel a los conceptos petrarquistas que reduce, en su rica alusividad potencial, a la univeridad de la poética castellana de cancionero. Coloma se aleja todavía más del texto original al hacer prevalecer las formas narrativas en detrimento de las descriptivas; los elementos abstractos frente a los concretos; la expresión real sobre la figurada, con el consiguiente deslucimiento, siquiera parcial, de la alegoria, consustancial a la "poética triunfal». Desviaciones que, significativamente, no se extienden a la omisión de la enumeración de las figuras mitológicas o alusiones eruditas, igualmente peculiares en el original petrarquesco, pero poética, al cabo, compartida por la estética cancioneril y, en general, por la lírica románica trecentista y cuatrocentista, no necesariamente petrarquesca:

Cosi parlaua; te gliocchi haue al ciel fissi

Diuotamente: poi mise in silentio

Quelle labra rosate, in fin ch"io dissi

Scylla, Mario, Neron, Gaios et Mezentio;

(Illicino, ed. cit., f' LX.v.)

Luego su boca cerró

y los ojos leuantó

al cielo el diuino gesto, do está colocado y puesto

a lo cual respondí yo.

«La muerte, lo que se lee de Silla, Mario y Nerón, Gayo, Mecencio...

(Coloma, ed. cit., p.14)

Al prescindir a menudo de una aproximación, verso por verso, al texto original (lo cual es sencillamente obvio) Coloma nos ofrece una versión más atenta al significado general que a la captación y traslación de los puntos culmninantes y esenciales de la poética petrarquesca (como hemos visto) o 
al mantenimiento de formas retóricas estructurales. Tal el caso de la repetición anafórica, rasgo retórico tan caro al petrarquismo y con el que se inicia el mismo Triunfo de Muerte en la versión de Illicino y Obregón y que Coloma, declina:

Quanti gia nella eta matura e sacra

Triumphi ornarono il glorioso colle.

Quanti prigion passar per la via sacra

Sotto il monarcha; che al suo tempo volle

Fare il mondo descriuere uniuerso:

Che il nome di grandeza aglialtri colle.

(Illicino, ed. cit., fo. L.r.)

Quantos triumphos adornaron, en la edad tan celebrada, el monte a do se mostraron por aquellos que passaron despoios la via sagrada Al tiempo que fue señor, monarca y emperador el que hizo numerar el mundo y pudo quitar nombres de grande valor.

(Coloma, ed. cit., p. 3.)

De igual modo que tiende a suprimir, conformado por una poética más enérgica, cortante y rápida en el discurrir del ritmo del verso, el uso profuso por parte de Petrarca del polisindeton con el que éste no sólo subraya, enfatizándola significativamente, la palabra precedida por la conjunción, sino que además remansa el ritmo del verso, toque de gracia de su endecasílabo:

V son hor le ricchezze? V son gli honori?

Et le gemme, e gli sceptri, e le corone?

(Illicino, ed. cit., fo. LV.r.)

¿a dónde están sus estados, riquezas, sceptros, coronas?

(Coloma, ed. cit., p. 7.) 
Es indudable que la traducción de Coloma, al trasladar los conceptos y en parte las palabras petrarquescas a un sistema poético distinto y aun opuesto como el cancioneril castellano, resiente las tensiones. Con todo, es también notorio que el relativo despego con que el traductor se enfrenta al texto original y la notable libertad con que conforma su propia traslación dotan a ésta, especialmente en relación al ejemplo de su predecesor Obregón, de un mayor dinamismo y coherencia con su propio arte poético cancioneril; propio, ya que no único, al menos después de que él mismo tiente, con más que con cierta maestría, el "arte toscano", sistema con el que, como advertimos, encabeza la segunda parte del Cancionero General de ubras nuevas (1554).

En la confrontación con las teorías de la traducción desarrolladas en la época, recientemente estudiadas por Ann J. Cruz ${ }^{54}$, justo en relación a los Triunfos, Traducidas por Obregón, Alvar Gómez y Hozes, pudiéramos decir que la traslación del Triunfo de la Muerte de Coloma consigue un equilibrio entre la fidelidad al texto primigenio en su sentido y la libertad (paradójicamente determinada por un sistema poético distinto al del original italiano, actuante en la formación poética y en los gustos del traductor) en la decantación métrica, en la elección del léxico y en el tratamiento morfosintáctico y estilístico; éste último notablemente transformado. No alcanza Coloma, por ahí, las altas cotas de perfección recomendadas por Leonardo Bruni al traductor en su De interpretatione recta (1426), que junto a la conversión del texto en el entendimiento, la mente y la voluntad exigía la reproducción del estilo del original ${ }^{\text {s' }}$; pero tampoco podemos calificar su traducción de una insípida traslación ad verbum, mera réplica literal, o totalmente ad sensum, atenta sólo al sentido, posibilidades estudiadas, junto a otras que atañen a la ornamentación, por Giannozzo Manetti ${ }^{5 / 2}$ y en parte recogidas por el Comento de Eusebio de Alfonso Madrigal, el Tostado, obra de notable difusión en el quinientos español ${ }^{57}$. Éste, ante el dilema de la traducción palabra por palabra (que llama interpretación) o la explicación del texto original (que denomina glosa), en su propia traducción del In Eusebium, se decanta por una solución complementaria ${ }^{5 x}$, es decir, una traducción con comentario, por la que, como hemos advertido, no optó Coloma a juzgar por el ejemplo publicado en el Cancionero general de obras

"The Trionfi in Spain: Petrarchist Poetics, Translation Theory, and the Castilian Vernacular in the Sixteenth Century cit.

"G. P. NORTON, artículo cit., pp. 189-190.

so De Interpretatione recta (1455), cit. por G. P. NORTON, Pp. 198-199.

"Ver R. I. Keightefy, Alonso Madrigal and the Chronaci Canones of Eusebius. "The Journal of Medieval and Renaissance Studies», 7 (1977), p. 246.

is R. I. Kelghtifley, pp. 244-245. 


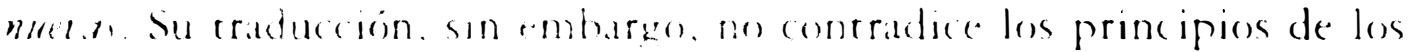
humanistas pues respetat el seritide ded texto origrinal, ofreciendo una ver-

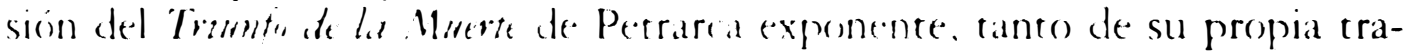

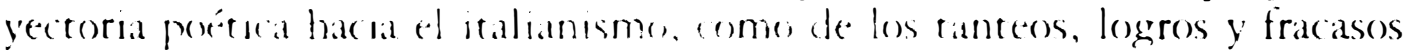

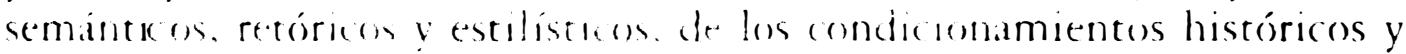

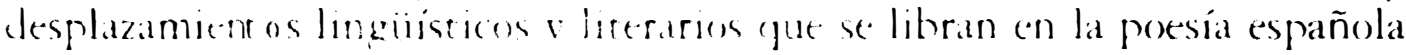
en la primera mitad de! stglo XVI, en especial en el segundo cuarto. De

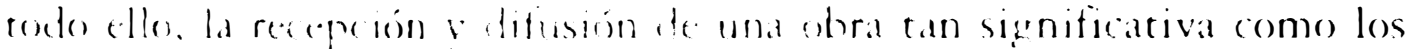

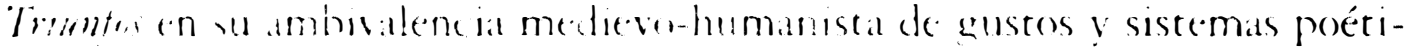
cos, con el ejemple de sus destintas craducciones puede constituirse en exponente de transecion, tanto haciat 6 m.is meridiano petrarquismo renacentista del canzontere como hacia la plena aclimaracion del italianismo en nuestra poesía.

\section{RISIME}

Cee article etude la traductoon du Timonto de la . Muerte de Pétrarque, rédigée

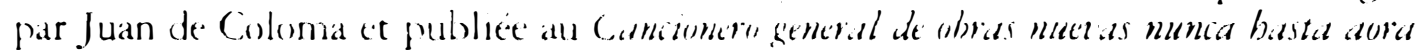

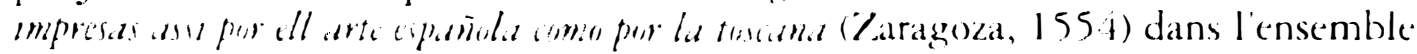

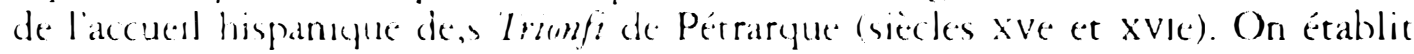
une comparaison entre les caractéristigues de cette traduction castillane et celles antéricures, dure a Obregon $(1512)$ et à Alvar Crómez (?), en ce qui concerne l'original italien. Dans l'article est évaluée aussi la traduction de Coloma à l’égard des théories de la traduction développées à l'époque de l'Humanisme et leur signification à l'occasion des changements poètiques entre la tradition de la chanson médiéval castillane et l'italianısante de la Renaissance.

\section{$\triangle M M A R Y$}

The article deals with the translation of Petrarchs Trangfo de la Muerte, writ-

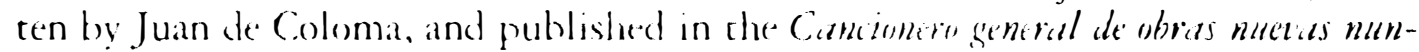

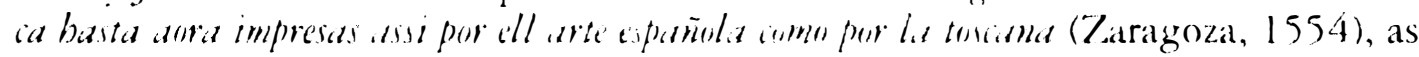
part of the Spanısh reception of Petrarchis Tritinfi (XV and XVI centuries). A comparison is made beeween the characteristics of this Spanish translation with the former ones, writen by Obregón (151.2) and Alvar (rome. (?), as far as the Italian original is concerned. The authoress of the article evaluates the Coloma translation in connecton with the theories on translation expounded during the humanistic period and the role of these theories in the context of the poetical changes that rook place between the Castilian traditional song of the Middle Ages and the Italianising Renaissance. 